\title{
Nondestructive Semistatic Testing Methodology for Assessing Fish Textural Characteristics via Closed-Form Mathematical Expressions
}

\author{
D. Dimogianopoulos ${ }^{1}$ and K. Grigorakis ${ }^{2}$ \\ ${ }^{1}$ Department of Automation Engineering, Piraeus University of Applied Sciences, 12244 Egaleo, Greece \\ ${ }^{2}$ Hellenic Centre for Marine Research, Institute of Marine Biology, Biotechnology \& Aquaculture, Agios Kosmas, \\ Hellinikon, 16777 Athens, Greece
}

Correspondence should be addressed to K. Grigorakis; kgrigo@hcmr.gr

Received 13 July 2016; Revised 7 January 2017; Accepted 12 January 2017; Published 30 January 2017

Academic Editor: Jorge Barros-Velázquez

Copyright (c) 2017 D. Dimogianopoulos and K. Grigorakis. This is an open access article distributed under the Creative Commons Attribution License, which permits unrestricted use, distribution, and reproduction in any medium, provided the original work is properly cited.

\begin{abstract}
This paper presents a novel methodology based on semistatic nondestructive testing of fish for the analytical computation of its textural characteristics via closed-form mathematical expressions. The novelty is that, unlike alternatives, explicit values for both stiffness and viscoelastic textural attributes may be computed, even if fish of different size/weight are tested. Furthermore, the testing procedure may be adapted to the specifications (sampling rate and accuracy) of the available equipment. The experimental testing involves a fish placed on the pan of a digital weigh scale, which is subsequently tested with a ramp-like load profile in a custommade installation. The ramp slope is (to some extent) adjustable according to the specification (sampling rate and accuracy) of the equipment. The scale's reaction to fish loading, namely, the reactive force, is collected throughout time and is shown to depend on the fish textural attributes according to a closed-form mathematical formula. The latter is subsequently used along with collected data in order to compute these attributes rapidly and effectively. Four whole raw sea bass (Dicentrarchus labrax) of various sizes and textures were tested. Changes in texture, related to different viscoelastic characteristics among the four fish, were correctly detected and quantified using the proposed methodology.
\end{abstract}

\section{Introduction}

Texture of fish is one of the principal characteristics indicative of its quality. Alterations of texture depend on many endogenous parameters including the species, sex, genetic background, and maturity. Texture is also affected by exogenous parameters such as the fish dietary history, aquaculture management in case of farmed fish, and fish handling during both slaughtering and postmortem $[1,2]$. Consequently, a straightforward evaluation of fish texture using a simple methodology is very important in order to obtain information regarding its quality, namely, its freshness and/or traceability.

The evaluation of fish texture by non-destructive techniques is mainly performed via specific organoleptic methods. These are essentially based on human sensory perception to evaluate texture and, thus, involve a degree of human subjectivity. Other alternatives for the nondestructive evaluation of texture include various indirect methods based on electric stimulation [3] or image analysis [4] of fish flesh. In fish industry, the most widely used nondestructive method is the so-called finger testing: a human subject exercises pressure on the fish flesh by finger in order to empirically evaluate its hardness and elasticity [1]. Then, in finger testing too, a high degree of human subjectivity is involved. It is obvious, hence, that any method achieving nondestructive measurement of such textural attributes in an objective (unaffected by human subjectivity) manner can prove valuable in industry.

By the past, effort has been invested in achieving the nondestructive assessment of fish hardness by means of commercially available instruments such as the Zwick hardness tester. The instrument applies static pressure on the tissue 
(fish fillet) and the hardness value is determined after dwell times of 1 or 3 seconds. Moreover, attempts to map hardness variations to changes in fish freshness were also carried out in Schubring [5]. In this case, Zwick tester measurements were correlated with sensory-perceived freshness (Quality Index Method (QIM)).

Other attempts to estimate variations in mechanical properties of fish (stiffness and viscoelastic characteristics) and relate them to its freshness level may be found in Grigorakis and Dimogianopoulos [6] and Dimogianopoulos and Grigorakis [7, 8]. In these cases, the fish was part of a vibrating structure, which was subjected to dynamic nondestructive loading. The variations in mechanical properties were estimated in an indirect manner: one approach was to monitor specific time points of the vibrating structure's response and look for changes or patterns caused by altered fish mechanical properties [6]. Another approach was to identify a stochastic model of the structure for each test case and analyze some key characteristics pointing to changes of fish mechanical properties $[7,8]$. This last work introduced modifications of the data processing in order to achieve reliable assessment of fish freshness, even when the tested fish were of different sizes/weights. Another extension of the previous scheme may be found in Grigorakis and Dimogianopoulos [9], where the variations in mechanical properties were correlated with the dietary history of the fish. Note that, in all cases, an indirect estimation of the variation in fish mechanical properties was performed. In other words, a closed-form formula explicitly relating the raw experimental data to the considered mechanical properties was not obtainable.

The proposed methodology innovates in two ways. First, it establishes the theoretical link between fish mechanical properties (stiffness and viscoelastic characteristics, which are relevant to its textural attributes) and the data collected from the fish nondestructive testing. This link consists in a closed-form mathematical formula, which relates the mechanical properties (and, hence, textural attributes) to experimental data in an explicit manner, independently of the fish weight. Second, it allows for a more flexible testing procedure via the use of a potentially semistatic loading profile. As will be shown in Materials and Methods, the testing procedure involves the application of a ramp-like load onto the fish, the slope of which is (to some extent) configurable according to the accuracy/sampling rate of the test equipment.

The benefit of these innovations lies in the fact that, contrary to all indirect methods found in previous works [6-9] and already commented upon, the user may now obtain effortlessly and reliably values for the fish mechanical properties: A given set of experimental data values may be used in conjunction with the closed-form mathematical formula to directly compute the stiffness and viscoelasticity characteristics via available curve-fitting algorithms. Moreover, the ramp-like loading profile has similarities to that used in the TPA initial loading phase. Then specific textural results obtained via the proposed method may be comparable to those from the TPA procedure, as is also highlighted in Results and Discussion.
The paper is organized as follows. In Introduction, a quick overview of the proposed methodology and its positioning with respect to other texture assessing schemes is given. In Materials and Methods, details are given on the tested fish, the testing procedure is explained, and the theoretical framework for the computation of the texture-related mechanical quantities is presented. In Results and Discussion, the application of the methodology on four whole raw sea bass (Dicentrarchus labrax) of various sizes/weights and texture is presented and commented upon. Finally, Conclusions presents some concluding remarks.

\section{Materials and Methods}

2.1. Fish. European sea bass (Dicentrarchus labrax) was previously farmed in the experimental facilities of the Hellenic Centre for Marine Research (HCMR). Fish were slaughtered according to the custom ice-water (2:1 volume/volume) immersion method, weighted, and stored covered with ice $\left(0^{\circ} \mathrm{C}\right)$ in a polystyrene box until measurement. Two batches of two fish each were sampled at 6-day interval, since sea bass sampled at similar intervals have been found to present different textural attributes both in TPA [10] and in previous studies of the authors $[6,8]$. Thus, fish stored in ice for one as well as for seven days were simultaneously available for testing. These fish, namely, $1 \mathrm{mc}$ and $1 \mathrm{md}$ and $7 \mathrm{mb}$ and $7 \mathrm{mc}$, respectively, were used to validate the proposed method.

\subsection{Principles of the Diagnostic Scheme's Operation}

2.2.1. Fish Testing Procedure. The testing procedure involves a digital weigh scale (electronic force gauge), which can measure force exercised on its pan throughout time and store these values in a file readable by a personal computer. The fish is placed on the scale pan and a load of ramplike profile is exercised on its dorsal area (Figure 1(a)). The application of a ramp-like load involves the use of a custommade simple structure (Figure 1(a)). It features a conical support into which a light empty rigid tube (similar to the glass ones found in chemistry labs) is free to slide on the vertical direction with minimal lateral play. The tube's end is placed on the fish dorsal area, so that its weight is directly exercised on the fish. Subsequently, the tube is gradually filled with water under constant flow, so that its weight increases in time at a ramp-like manner. Hence, the fish may admit a ramp-like load on its dorsal area with the ramp slope being controlled via the water flow selected by the user. If water flow is reliably controlled by the user, the ramp-like loading is relatively well reproducible and may admit profiles ranging from the semistatic (a ramp increasing very slowly) to a dynamic one (a rapidly increasing ramp). Note that the choice of the ramp profile is a trade-off between successfully exciting the fish for exposing its behavior under load and satisfying the limitations of the available equipment. This trade-off will be further discussed in Results and Discussion, but in the current case the successfully tested loading rates ranged from as low as 100 up to 250 gr of water per second. In general, a rate of ca 200-250 gr of water per second (corresponding to 


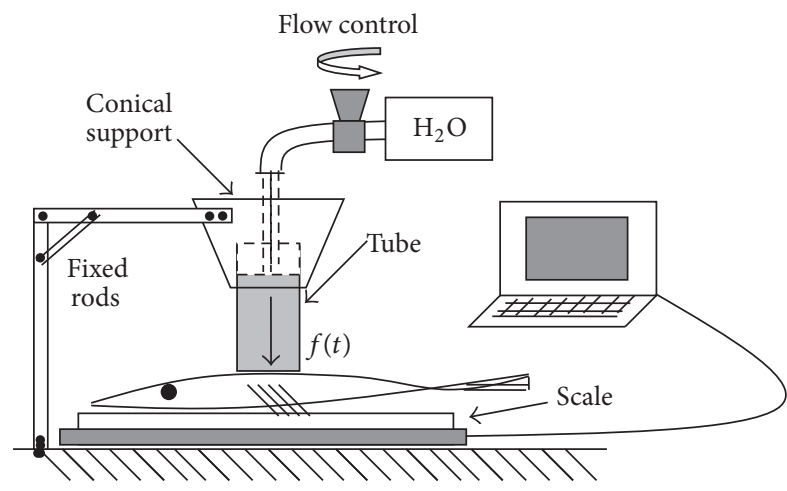

(a)

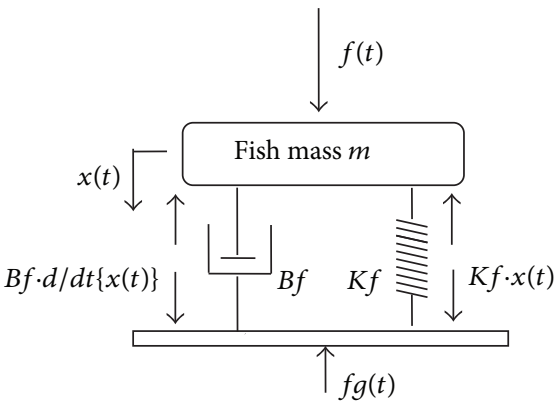

(b)

FIGURE 1: (a) The testing structure involving the fish, scale, conical tube support secured by fixed rods, and water supply; (b) the Kelvin-Voigt model of the fish.

instant wide opening of the water supply) was selected mainly because it was easily and consistently reproducible at each test run.

The pan's response in form of reactive force $f g(t)$ on the loaded fish is measured by the digital scale, and its values throughout time (hereafter referred to as response data) are stored in a file. This typical loading was repeated 40 times for each of the four tested fish. Given that the loading was almost identical for all fish, differences among the response data $f g(t)$ can only be attributed to textural changes among the fish tested, since these are the only alterable system parts. The test data were then processed by means of an algorithm described in the following section.

\subsubsection{System Modeling and Derivation of Reactive Force Equa-} tion. The fish, placed on the pan of the weigh scale, is represented by the Kelvin-Voigt model shown in Figure 1(b). In essence, the Kelvin-Voigt model is a mechanical analogue of the fish formed by a solid spring element in parallel with a damping element. It provides a useful means of representing complex viscoelastic/rheological behavior in foods. Other more complex approaches also involving specific combinations of spring and damping elements include the Maxwell model (in simple or generalized versions), the Zener or Standard Linear Solid (SLS) model, and the Burger model (see [11], ch. 5).

According to the Kelvin-Voigt model, the fish mass $m$ is suspended by a spring of constant $K f$ and a damping element of constant $B f$. The spring element is considered to obey Hooke's law, meaning that when compressed by $x$ meters it reacts by means of an opposite (to the direction of compression) force equal to $K f \cdot x$ Newtons. The spring element essentially represents the stiffness/elasticity of fish flesh and describes its aptitude to withstand small static load without suffering permanent deformation. The damping element is considered to obey Newton's law, meaning that when compressed at a speed of $v \mathrm{~m} / \mathrm{sec}$ it reacts by means of an opposite (to the direction of compression) force equal to $K f \cdot v$ Newtons. The damping element essentially represents the viscoelastic behavior of the fish flesh: in other words, its aptitude to exhibit resistance when deformed under dynamic loading.

In the current case, applying an external load $f(t)$ onto the fish of mass $m$ causes the spring to respond by applying a force equal to $K f \cdot x(t)$ onto the mass $m$ and onto the pan, as shown in Figure 1(b). The damping element also responds by an applying force equal to $B f \cdot d / d t\{x(t)\}$ onto the mass $m$ and onto the pan, as shown in Figure 1(b). Note that $x(t)$ and $d / d t\{x(t)\}$ stand for the displacement from the equilibrium point (which is the initial position admitted due to the fish's own weight) and the speed of deformation, respectively.

The weigh scale pan is solidly placed on the ground and is not deformable, thus forming one group upon which the fish is set. That means that the group of pan-and-ground receives the forces from the spring and damping elements and responds with an equal but opposite force $f g(t)=K f \cdot x(t)+$ $B f \cdot d / d t\{x(t)\}$, as shown in Figure 1(b). Applying Newton's law of motion on the mass $m$ yields

$$
-K f \cdot x(t)-B f \cdot \frac{d}{d t} x(t)+f(t)=m \cdot \frac{d^{2}}{d t^{2}} x(t)
$$

or, by suitably rearranging terms,

$$
m \cdot \frac{d^{2}}{d t^{2}} x(t)+B f \cdot \frac{d}{d t} x(t)+K f \cdot x(t)=f(t) .
$$

In (2), $d^{2} / d t^{2}\{x(t)\}$ is the acceleration of mass $m$ during loading. This differential equation may be handled by means of system control engineering principles [12]. Applying Laplace transform in (2), one has

$$
m \cdot s^{2} \cdot X(s)+B f \cdot s \cdot X(s)+K f \cdot X(s)=F(s)
$$

with $X(s)$ and $F(s)$ being the displacement $x(t)$ and force $f(t)$ signals in the Laplace domain. Recall that, in the Laplace domain, the product $s \cdot X(s)$ corresponds to $d / d t\{x(t)\}$ [the first derivative of $x(t)]$ in the time domain, whereas $s^{2} \cdot X(s)$ corresponds to $d^{2} / d t^{2}\{x(t)\}$. The transfer function $X(s) / F(s)$ is then easily obtained as

$$
\frac{X(s)}{F(s)}=\frac{1}{m \cdot s^{2}+B f \cdot s+K f} .
$$


As stated before, it holds that $f g(t)=B f \cdot d / d t\{x(t)\}+K f \cdot x(t)$, meaning that in the Laplace domain

$$
F g(s)=B f \cdot s \cdot X(s)+K f \cdot X(s) .
$$

Combining (4) and (5) yields

$$
\begin{aligned}
\frac{(B f \cdot s+K f) \cdot X(s)}{F(s)} & =\frac{B f \cdot s+K f}{m \cdot s^{2}+B f \cdot s+K f} \Longrightarrow \\
\frac{F g(s)}{F(s)} & =\frac{B f \cdot s+K f}{m \cdot s^{2}+B f \cdot s+K f},
\end{aligned}
$$

where $F g(s) / F(s)$ is the transfer function between the loading imposed on the fish and the response force from the pan. Performing standard algebra on (6), one has

$$
\begin{aligned}
\frac{F g(s)}{F(s)} & =\frac{B f \cdot s+K f}{m \cdot s^{2}+B f \cdot s+K f} \\
& =\frac{B f / m \cdot s+K f / m \pm s^{2}}{s^{2}+B f / m \cdot s+K f / m} \\
& =1-\frac{s^{2}}{s^{2}+B f / m \cdot s+K f / m}
\end{aligned}
$$

or

$$
\begin{aligned}
F g(s)= & F(s)-\frac{m}{K f} \cdot \frac{K f / m}{s^{2}+B f / m \cdot s+K f / m} \cdot s^{2} \\
& \cdot F(s) .
\end{aligned}
$$

Equation (8) states that the reactive force $F g(s)$ expressed in the Laplace domain (or equally $f g(t)$ in the time domain) is equal to the known (since user-defined) external loading minus the response to input $s^{2} \cdot F(s)$ from the second-order dynamic system $G(s)$ defined as

$$
G(s)=\frac{m}{K f} \cdot \frac{K f / m}{s^{2}+B f / m \cdot s+K f / m}
$$

with the static gain of $G(s)$ being equal to $m / K f$. Then, provided that the response of $G(s)$ to input $s^{2} \cdot F(s)$ can be calculated, the reactive force is also easily derived. Fortunately, it is easy to compute the response of $G(s)$ for a multitude of inputs, since such second-order systems have been extensively studied in [12].

Hence, one may proceed by defining the characteristic quantities of second-order systems [12], namely, the damping factor $\zeta$ and natural frequency $\omega n$, as follows:

$$
\begin{aligned}
\zeta & =\frac{1}{2} \cdot \frac{B f}{\sqrt{K f \cdot m}}, \\
\omega n & =\sqrt{\frac{K f}{m}} \Longrightarrow \\
G(s) & =\frac{1}{\omega n^{2}} \cdot \frac{\omega n^{2}}{s^{2}+2 \cdot \zeta \cdot \omega n \cdot s+\omega n^{2}} .
\end{aligned}
$$

Using (8), (9), and (10) one has

$$
\begin{aligned}
F g(s)= & F(s)-\frac{1}{\omega n^{2}} \cdot \frac{\omega n^{2}}{s^{2}+2 \cdot \zeta \cdot \omega n \cdot s+\omega n^{2}} \cdot s^{2} \\
& \cdot F(s) .
\end{aligned}
$$

Given that the external load $f(t)$ applied onto the fish is characterized by a ramp-like profile, it should hold that $f(t)=$ $A \cdot t$. This states that the force imposed on the fish increases linearly with time $t$ and is characterized by a slope equal to $A$. Then, from Laplace Transform Tables in [12], the Laplace transform of $f(t)$ is equal to $F(s)=A / s^{2}$. Introducing $F(s)=$ $A / s^{2}$ into the part of (11) related to $G(s)$ yields

$$
\begin{aligned}
F g(s) & =F(s)-\frac{1}{\omega n^{2}} \cdot \frac{\omega n^{2}}{s^{2}+2 \cdot \zeta \cdot \omega n \cdot s+\omega n^{2}} \cdot A \\
& =F(s)-G(s) \cdot A .
\end{aligned}
$$

Equation (12) is very important since it states that the reactive force is equal to the known ramp-like loading imposed on the fish minus the also known impulse response of system $G(s)$, which is readily available in [12]. Applying inverse Laplace transform to (12) yields

$$
f g(t)=A \cdot t-A \cdot[\text { impulse response of } G(s)] .
$$

From [12], the impulse response $g(t)$ of the second-order system $G(s)$ in (13) is available as

$$
g(t)=\frac{1}{\omega n \cdot \sqrt{1-\zeta^{2}}} \cdot e^{-\zeta \cdot \omega n \cdot t} \cdot \sin \left(\omega n \cdot \sqrt{1-\zeta^{2}} \cdot t\right) .
$$

Combining (13) and (14), it follows that

$$
\begin{aligned}
f g(t)= & A \cdot t-A \cdot \frac{1}{\omega n \cdot \sqrt{1-\zeta^{2}}} \cdot e^{-\zeta \cdot \omega n \cdot t} \\
& \cdot \sin \left(\omega n \cdot \sqrt{1-\zeta^{2}} \cdot t\right)
\end{aligned}
$$

with $\zeta$ and $\omega n$ defined in (10) and $A$ being the constant ramp slope. Note that the effect of fish mass is included in $\zeta$ and $\omega n$ as defined in (10), meaning that expression (15) may be used with tested fish of any mass. The constant $A$ may be defined by placing the empty tube on the pan, filling it with water using a predefined water flow value, and examining the tangent of the recorded response force $f g(t)$ in time.

A brief outline of the steps required for the computation of constants $K f$ and $B f$ in a direct manner based on (10) and (15) is given as follows:

(i) A fish placed on the pan is tested with a known ramplike load $f(t)=A \cdot t$ and values of response $f g(t)$ are recorded for each test run.

(ii) Each of the values $f g(t)$ recorded during the experimental test run is considered as the value given by (15) at time $t$ provided that the suitable (but unknown) values of $\zeta$ and $\omega n$ are used. Considering all available time instants $t$, a curve of $f g(t)$ in time is formed. 
(iii) Using curve-fitting algorithms (commonly available in software packages like MATLAB(R)), suitable values for $\zeta$ and $\omega n$ may be computed so that (15) reproduces the previous curve of $f g(t)$ in time.

(iv) By means of (10), the constants $K f$ and $B f$ may easily be computed.

2.2.3. Statistical Analysis. Given that four fish are tested by means of the proposed methodology, four sets of measurements are involved. Each set includes forty data values of reactive force and consequently forty values for $K f$ and $B f$ obtained via (10) and (15). The statistical comparison of $K f$ and $B f$ data starts with performing statistical control of their distributions. In case these are found to be normal, comparisons are processed via ANOVA, whereas in case of nonnormal distributions the nonparametric counterpart of ANOVA, namely, the Kruskal-Wallis method, is used.

\section{Results and Discussion}

3.1. Results Obtained via the Proposed Methodology. The postmortem spoilage process has indeed a significant effect on the mechanical properties of fish. $K f$ and $B f$ values of the four studied fish appear in Table 1 . The data sets involving $K f$ and $B f$ values underwent normality testing, and, due to nonnormal distributions, results were statistically processed by Kruskal-Wallis method for the detection of potential differences between $K f$ 's and $B f$ 's of individual fish. Kruskal-Wallis method distinguished $B f$ 's between different fish specimens $(p=0.005)$ but not $K f(p=0.843)$. These results are coherent with those obtained in Grigorakis and Dimogianopoulos [6], even though the fish at that study were tested under purely dynamic loading and the stiffness and viscoelasticity were computed in a different (indirect) manner.

The post hoc grouping of the four fish appears in Table 1. Based on this, it appears that the values for $B f$ of two 1-day fish do not differ, while the values for $B f$ of 7-day specimens are statistically significant compared to those of the $1 \mathrm{mb}$ fresh fish. Although the mean value of $1 \mathrm{mc}$ is higher than those of the two 7-day specimens, it can be grouped together with them, as well as with $1 \mathrm{md}$. This implies that $B f$ is reduced in 7-day fish when compared to 1-day ones, with the difference in their mean values is notable. The main obstacle in exposing this difference in mean is due to the high standard deviations in data sets containing $B f$ and $K f$ values.

The values obtained for the standard deviation point to the current limitation of the proposed methodology, which is primarily related to a purely technical matter: the way that the load is applied onto the fish. The main concern is to ensure that water filling of the tube is done in the same way under all circumstances, with water level rising in a steady manner. The latter is, currently, the subject of fine-tuning efforts towards obtaining better accuracy and repeatability of testing conditions. Such improvements would result in easier discrimination between samples of different texture properties, like, in this case, between samples of 1 day and 7 days of ice storage.
TABLE 1: $K f$ and $B f$ (means standard deviations) of the four studied fish specimens. Different letters (a and b) stand for statistically significant differences $(p<0.05)$.

\begin{tabular}{lcc}
\hline Fish & $K f$ & $B f$ \\
\hline $1 \mathrm{mc}$ & $6347 \pm 1270$ & $3.43 \pm 2.11^{\mathrm{ab}}$ \\
$1 \mathrm{md}$ & $6285 \pm 1292$ & $4.24 \pm 2.32^{\mathrm{a}}$ \\
$7 \mathrm{mb}$ & $6573 \pm 1827$ & $2.79 \pm 2.18^{\mathrm{b}}$ \\
$7 \mathrm{mc}$ & $6535 \pm 2012$ & $2.07 \pm 1.56^{\mathrm{b}}$ \\
\hline
\end{tabular}

Finally, given that the total reactive force includes forces due to both $K f$ and $B f$ (see (5)), care should be taken when choosing the ramp loading profile. As stated in Fish Testing Procedure of Materials and Methods, the ramp load profile cannot be arbitrarily slow in practice. This is due to the fact that a very slowly increasing ramp will cause reaction mainly from the spring with constant $K f$ : reaction of the viscoelastic element with constant $B f$ will only be important if the speed of loading is high. If the latter is close to zero, meaning that an almost static ramp is applied, then computing the constant $B f$ becomes harder, due to its insignificant contribution to the reactive force data. In this case, the choice of a weigh scale of higher precision is necessary. Nonetheless, the proposed method provides the necessary theoretical basis for using relatively slow (semistatic) ramp profiles, under the condition that the equipment features the precision required for recording small forces accurately and consistently.

3.2. Connection with Other Studies. It is known that fish, and sea bass in particular, becomes softer and less elastic after rigor mortis resolution, which occurs in about 6 days postmortem when stored in ice [13-15]. This sensory observation is in agreement with findings of the current study, namely, the reduced viscoelasticity of the 7 th day specimens. This is also the reason for the choice of 1-day fish (which are supposed to have entered rigor mortis) and 7-day fish (which are just entering postrigor state). The present results are also in agreement with those derived for the same species by TPA [10]. In fact, Molina et al. [10] have noted a marked difference in hardness for a TPA performed (among others) on 2-dayold and 7-day-old sea bass fillets, achieving compression of $25 \%$ at a probe speed of $1 \mathrm{~mm} / \mathrm{sec}$. The same authors similarly found difference between freshly harvested (0-day-old) and 2-day-old fish, with the latter exhibiting higher hardness during rigor mortis evolution, but failed to detect hardness difference between freshly harvested prerigor (0-day-old) and 7-day-old, postrigor state fish.

It is important to note that the hardness force measured corresponds to the total reactive force in the proposed methodology in (5), and hence it includes forces due to both $K f$ and $B f$ elements. Given that $K f$ is found to admit comparable values for all fish tested, it seems that reductions in $B f$ could explain the hardness reduction between 2-dayold and 7-day-old fish in Molina et al. [10], even though comparisons of raw force values are not straightforward: the TPA results are in terms of force values, whereas the currently proposed methodology computes constants $K f$ and $B f$ of 
spring and damping elements, which in turn produce the forces.

In [16], TPA was applied to sea bass fillets of various postmortem time intervals. The TPA settings (probe and compression rate) were similar, except for the probe speed being twice as big $(2 \mathrm{~mm} / \mathrm{sec})$. The results presented a hardness pattern with some similarities to the current study. These include an initial increase up to day 4 postmortem and subsequent decease from day 4 of ice storage to day 6 (and thereafter), thus providing further evidence on the validity of results in Molina et al. [10] and those presented herein.

Note also that since the first sampling occurred in day 1 postmortem, the onset of rigor mortis is in progress according to findings in Molina et al. [10]. Due to this fact, some differentiation among such individual fish is possible, which, in turn, could explain why the two 1-day samples showed different statistical grouping with respect to the 7-day fish.

\section{Conclusions}

A nondestructive methodology using semistatic test loading of fish in order to analytically compute textural attributes (relevant to fish stiffness and viscoelasticity) has been presented. The testing procedure may be (to some extent) configured in order to suit the specifications (sampling rate and accuracy) of the available equipment. Under conditions, this loading procedure may also highlight similarities between specific textural results obtained via the proposed method and TPA. The testing involves a fish placed on the pan of a digital weigh scale, a ramp-like load applied onto it by means of a simple custom-made installation, and the recording of reactive force measured by the scale throughout time. It is demonstrated that the reactive force depends upon the texture-related mechanical quantities of the fish via a closedform mathematical formula. This is, hence, used along with the data collected for a tested fish in order to compute the texture-related mechanical quantities in a rapid and efficient manner. Four whole raw sea bass (Dicentrarchus labrax) of various sizes/weights and texture were tested. Changes in texture, related to different viscoelastic characteristics among the four fish, were correctly detected and quantified using the proposed methodology.

\section{Disclosure}

The views expressed in this work are the sole responsibility of the authors and do not necessarily reflect the views of the European Commission.

\section{Competing Interests}

The authors declare that there are no competing interests regarding the publication of this paper.

\section{Acknowledgments}

This study was funded by the European Union (ARRAINA, FP7-KBBE-2011-5-288925, Advanced Research Initiatives for Nutrition and Aquaculture).

\section{References}

[1] Z. Coppes-Petricorena, "Texture measurements in fish and fish products," in Handbook of Seafood Quality, Safety and Health Applications, pp. 130-138, Wiley-Blackwell, 2010.

[2] K. Grigorakis, "Effects of nutrition and aquaculture practices on fish quality," in Handbook of Seafood Quality, Safety and Health Applications, M. K. Alasalvar Cesarettin, S. Fereidoon, and W. Udaya, Eds., pp. 82-95, 2010.

[3] M. Singer and J. Kutzko, "Method and system for the determination of palatability," Google Patents, US 11/548,003, 2007.

[4] S. A. Jensen and P. Haagensen, "A method of measuring meat texture," Google Patents, vol. EP0290540B1, Lumetech A.S., 1993.

[5] R. Schubring, "Texture measurement on gutted cod during sotrage in ice, using a hand-held instrument," Information Fischwirtschaft Fischereiforschen, vol. 49, pp. 25-27, 2002.

[6] K. Grigorakis and D. Dimogianopoulos, "Cost-effective and nondestructive textural assessment of fish freshness via system identification principles," Journal of Texture Studies, vol. 41, no. 4, pp. 492-510, 2010.

[7] D. Dimogianopoulos and K. Grigorakis, "A statistical modelbased diagnostic scheme for cost-effective determination of freshness in fish industry," in Proceedings of the 19th Mediterranean Conference on Control and Automation (MED '11), pp. 1409-1414, IEEE, Corfu Island, Greece, June 2011.

[8] D. Dimogianopoulos and K. Grigorakis, "Nondestructive textural assessment of fish freshness: a stochastic model-based approach robust to fish size variations," Journal of Texture Studies, vol. 45, no. 4, pp. 274-287, 2014.

[9] K. Grigorakis and D. Dimogianopoulos, "Non-destructive stochastic model-based detection of diet-induced alterations in fish texture," Journal of Food Measurement \& Characterization, vol. 6, no. 1-4, pp. 35-47, 2012.

[10] B. Molina, M. I. Sáez, T. F. Martínez, J. L. Guil-Guerrero, and M. D. Suárez, "Effect of ultraviolet light treatment on microbial contamination, some textural and organoleptic parameters of cultured sea bass fillets (Dicentrarchus labrax)," Innovative Food Science \& Emerging Technologies, vol. 26, pp. 205-213, 2014.

[11] J. F. Steffe, Rheological Methods in Food Process Engineering, Freeman Press, East Lansing, Mich, USA, 1996.

[12] R. C. Dorf and R. H. Bishop, Modern Control Systems, Pearson Education, Upper Saddle River, NJ, USA, 2010.

[13] N. Erkan, "Freshness and quality of aquacultured sea bass (Dicentrarchus labrax) and sea bream (Sparus aurata) stored in ice," Archiv fur Lebensmittelhygiene, vol. 58, no. 3, pp. 98-106, 2007.

[14] N. Erkan and Ö. Özden, "Gutted and un-gutted sea bass ( Dicentrarchus Labrax ) stored in ice: influence on fish quality and shelf-life," International Journal of Food Properties, vol. 9, no. 2, pp. 331-345, 2006.

[15] B. M. Poli, G. Parisi, G. Zampacavallo et al., "Quality outline of European sea bass (Dicentrarchus labrax) reared in Italy: shelf life, edible yield, nutritional and dietetic traits," Aquaculture, vol. 202, no. 3-4, pp. 303-315, 2001.

[16] D. Majolini, A. Trocino, M. Tazzoli, and G. Xiccato, "Evolution of European sea bass (Dicentrarchus labrax) freshness during storage," Italian Journal of Animal Science, vol. 8, no. 3, pp. 282284, 2009. 

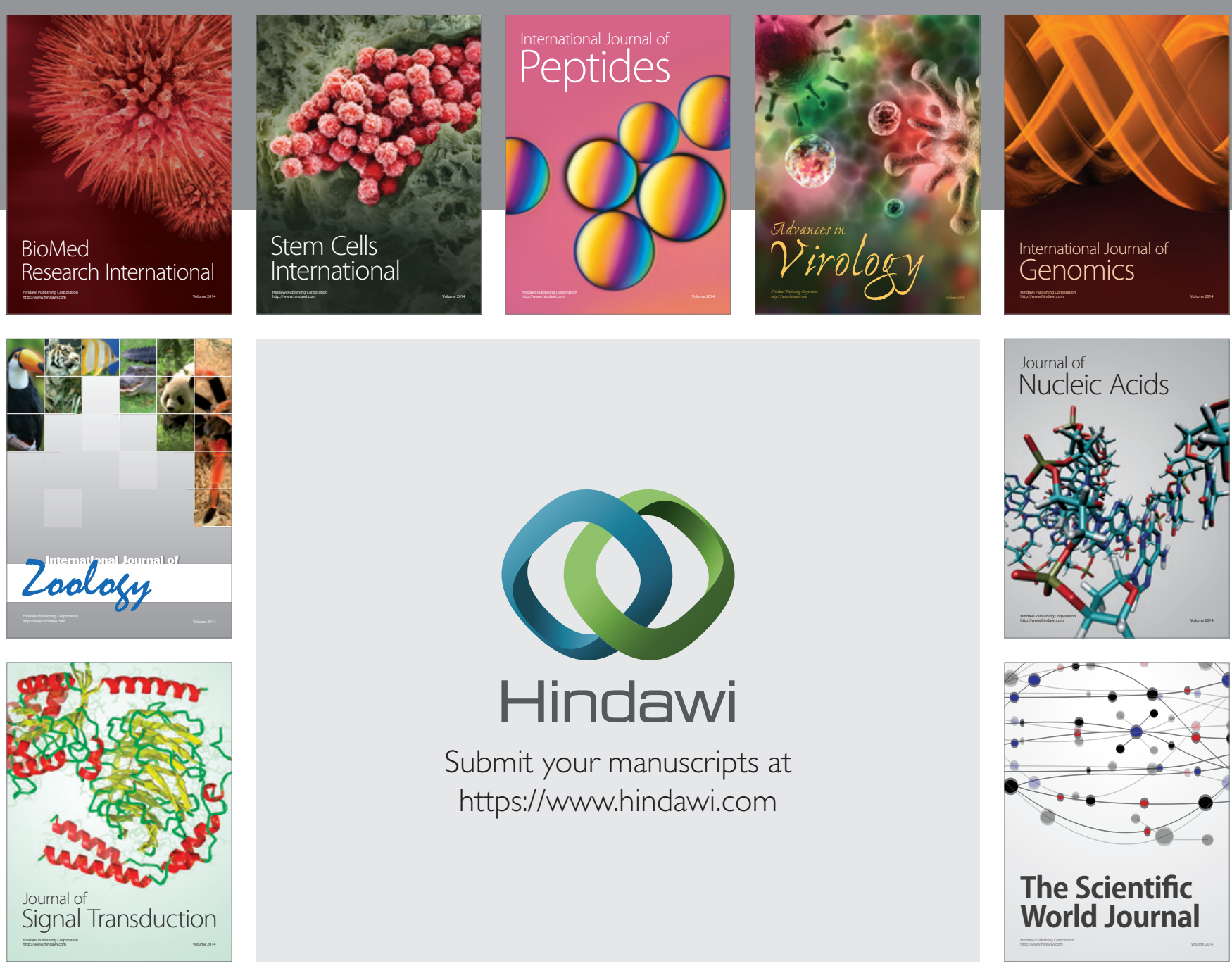

Submit your manuscripts at

https://www.hindawi.com
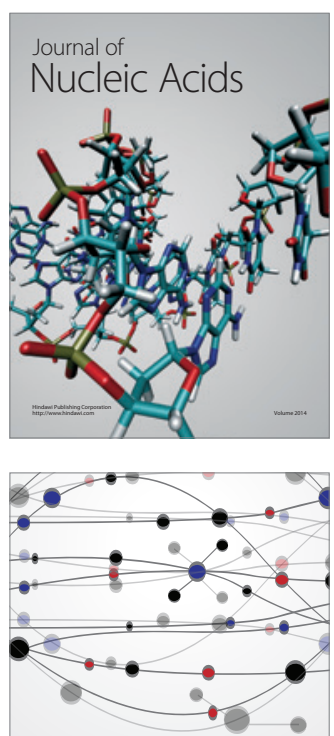

The Scientific World Journal
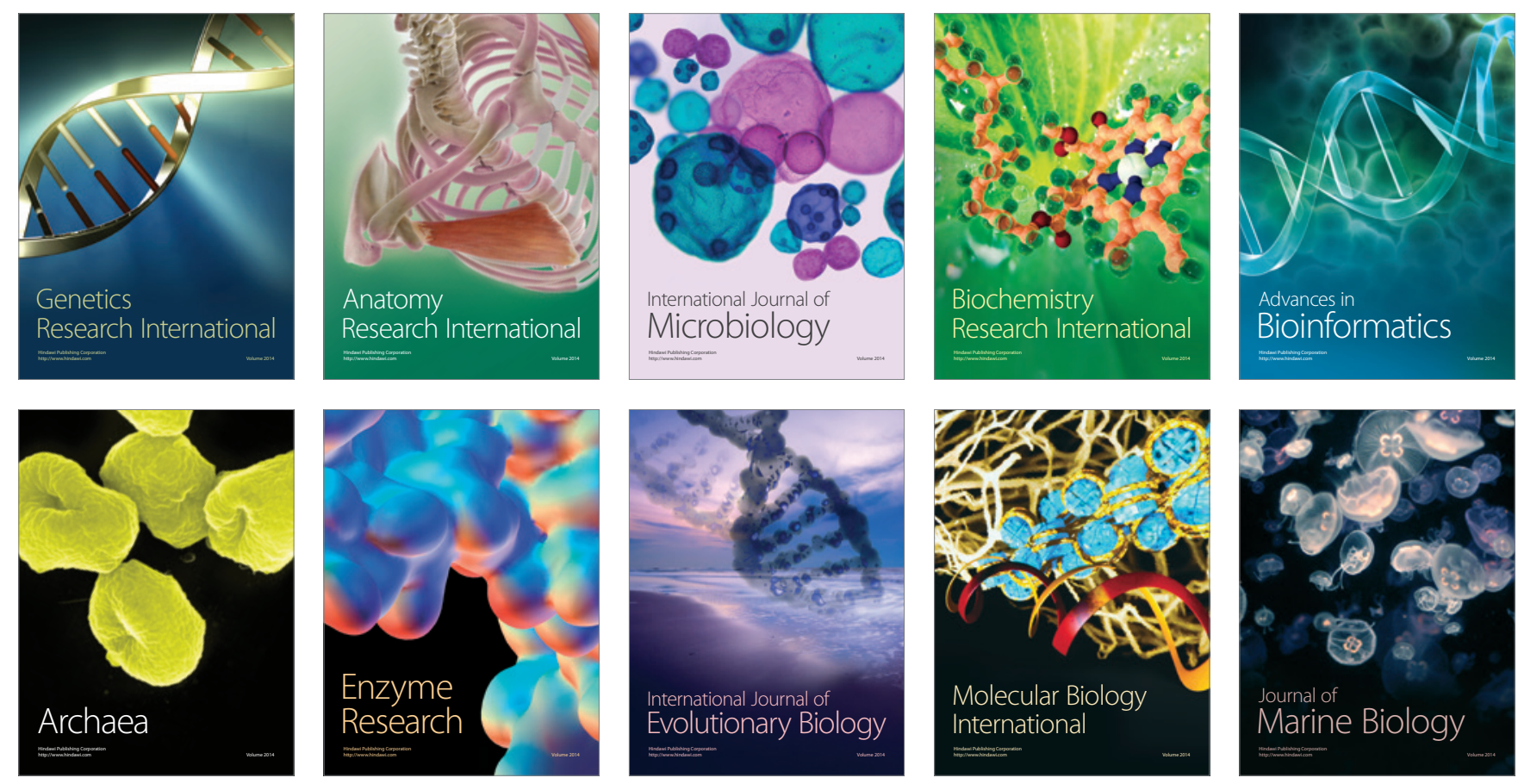